\title{
Heart Model A.I. Three-Dimensional Echocardiographic Evaluation of Left Ventricular Function and Parameter Setting
}

\author{
Yuan-Yuan Xing ${ }^{1,2}$ \\ Hong-Yuan Xue ${ }^{2}$ \\ Yu-Quan $Y e^{1,2}$
}

'Hebei Medical University, Shijiazhuang, Hebei, 0500 I7, People's Republic of China; ${ }^{2}$ Department of Ultrasound, Hebei General Hospital, Shijiazhuang, 05005I, Hebei, People's Republic of China
Correspondence: Yu-Quan Ye Department of Ultrasound, Hebei General Hospital No. 348 Heping West Road, Shijiazhuang, Hebei, 05005I, People's Republic of China Email yuquanyeyy@163.com
Objective: This study aims to explore the feasibility of HeartModel A.I. (HM) threedimensional echocardiography (3DE) to assess left ventricular function and discover suitable border parameter settings.

Methods: A total of 113 patients that underwent echocardiography in our hospital were eligible for inclusion. The HM 3DE (HM method) and conventional 3DE (3D method) were used to analyze echocardiography images. The HM was set to different border settings (end-diastolic [ED] and end-systolic [ES] settings) to assess different left ventricular systolic function parameters including left ventricular end diastolic volume (LVEDV), left ventricular end systolic volume (LVESV), and left ventricular ejection fraction (LVEF), and left ventricular diastolic function parameters including maximal left atrium volume $\left(\mathrm{LAV}_{\mathrm{MAX}}\right)$. All of these parameters were evaluated using the HM method and then compared with the 3D method.

Results: The differences in LVEDV, LVESV, and LVEF measured with different HM border settings were statistically significant $(\mathrm{P}<0.05)$ and were strongly correlated with the $3 \mathrm{D}$ method. For LVEF, the reading using the HM method with ED and ES $=70$ and 30 showed the best agreement with the 3D method, and the difference in the readings was not statistically significant $(\mathrm{P}>0.05)$. For LVEDV and LVESV, the reading using the HM method with ED and ES $=40$ and 20 showed the best agreement with the 3D method, but the difference in the readings was statistically significant $(\mathrm{P}<0.05)$. The measurements taken using the HM method were more reproducible than those taken using the $3 \mathrm{D}$ method $(\mathrm{P}<0.05)$. The measurement time when using the HM method was significantly less than the $3 \mathrm{D}$ method $(\mathrm{P}<0.05)$. In terms of $\mathrm{LAV}_{\mathrm{MAX}}$, the correlation between the HM and $3 \mathrm{D}$ methods was strong, but the requirements for agreement were not satisfied.

Conclusion: Evaluation of the left ventricular function using HM 3DE is feasible, saves time, and is reproducible. To assess the left ventricular function, the border parameter setting of $\mathrm{ED}$ and $\mathrm{ES}=70$ and 30 provided the best fit for the Chinese population.

Keywords: three-dimensional echocardiography, endocardial border delineation, left ventricular function, left ventricular volume

\section{Introduction}

In 2016, the European Association of Echocardiography updated its guidelines for the diagnosis and treatment of acute and chronic heart failure ${ }^{1}$ to include a more detailed classification of heart failure on the basis of a series of clinical symptoms, signs, and abnormal laboratory indicators. When considering ejection fraction (EF), heart failure is divided into heart failure with reduced EF ( $\leq 40 \%)$, median EF (41-49\%), and preserved EF ( $\geq 50 \%$ ). Therefore, accurately, quickly, and reproducibly evaluating 
cardiac function, especially left ventricular function, is a critical issue that urgently needs to be solved. The guideline clearly states that echocardiography is the most widely used method for the evaluation of heart function, as it provides pivotal information about chamber volume and ventricular systolic and diastolic functioning. The role it plays in the diagnosis and treatment of heart disease is of paramount importance because it is portable and noninvasive, and the results are reproducible. The accuracy of M-mode and twodimensional echocardiography has been questioned owing to the geometric assumptions and foreshortened views associated with these techniques. Although the results of conventional three-dimensional echocardiography (3DE) have been proved to be closely correlated with the gold standard cardiac magnetic resonance imaging (CMR), and 3DE has been recommended as a diagnostic tool for evaluating left ventricular functioning, ${ }^{2,3}$ it is complicated to operate, time consuming, and highly dependent on the proficiency of the operator, which means that this accurate and reproducible methodology is unsuitable for daily clinical practice., ${ }^{4,5}$

Artificial intelligence is an invaluable tool that has been used in many areas. Left ventricular function is of the utmost importance for diagnostic and prognostic considerations. HeartModel A.I. (HM) is a new automated left ventricular volume quantitative technology. Its principle is based on the segmentation algorithm of the Anatomical Intelligent Ultrasound model and combines artificial intelligence and conventional 3DE to automatically measure the left ventricular volume and function. In 2016, Tsang et $\mathrm{al}^{6}$ attempted to use HM to assess left ventricular systolic function and obtained gratifying results. Subsequently, a multi-center prospective validation study conducted by Medvedofsky et $\mathrm{al}^{7}$ further confirmed the usefulness of this method. HM provides the end-systolic (ES) and end-diastolic (ED) border parameter setting options (ED and ES), but until now, few studies have examined the optimal settings for these parameters. ${ }^{8,9}$ Therefore, this study examines the Chinese adult population and applies different border parameter settings for HM. HM 3DE (HM method) is compared with conventional 3DE (3D method) to explore the feasibility of the HM method for measuring left ventricular systolic and diastolic function and determine reasonable parameter settings. We found that the LVEF, LVEDV, and LVESV measured with different HM border settings showed a good correlation with the results of the conventional 3DE, and the settings of ED and ES $=70$ and 30 and 70 and 40 showed the best correlation with conventional 3DE. The difference in correlation between the different parameters showed no real practical significance owing to the number of confounding factors.

\section{Materials and Methods}

\section{Patients}

This study selected consecutive outpatients and inpatients that underwent echocardiography at our institution (a tertiary hospital) from August 2019 to April 2020. Heart disease was excluded in all patients by medical history, electrocardiogram, and echocardiogram. The exclusion criteria were: (1) non-sinus rhythm; (2) congenital heart disease and other obvious abnormalities in the heart structure; and (3) more than two segments out of 17 segments with poor endocardial visualization. This study was approved by the Medical Ethics Committee of our hospital, and informed consent was obtained from all the selected participants. A total of 160 subjects were collected and 47 subjects (29.3\%) were excluded.

\section{Image Acquisition and Analysis}

Imaging was performed using the Philips EPIQ 7C scanner and X5-1 transducer (1-5 MHz) equipped with $\mathrm{HM}$ and 3D software for measuring cardiac function. The echocardiographer (the doctor who checks patients, acquires images, and makes the analyses) created a new case and entered the basic information for each subject. The patients were placed in the left lateral decubitus position and connected to the electrocardiogram. The gain, depth, sector width, time gain compensation, and other conditions were adjusted to make the display of the endocardial boundaries clear.

The X5-1 probe was placed on the apex of the heart to obtain a clear apical four-chamber heart view, the HM ACQ was started, and the imaging depth and sector width were adjusted to clearly display the endocardial boundaries. The patients were asked to hold their breath, and the Acquire1 key was pressed to collect $3 \mathrm{D}$ images of the left ventricle over five cardiac cycles. The HM post-processing software was started, which automatically identifies the ED and ES phases of the cardiac cycle, and the HM border settings for ED and ES were set to 40 and 20, 60 and 30, 60 and 50, 70 and 30, 70 and 40, 74 and 68, 80 and 40, 90 and 50, and 100 and 50. The software automatically recognized the end-systole and enddiastole endocardium and obtained the LVEDV, LVESV,

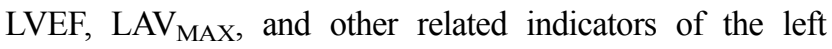
ventricular systolic and diastolic function (see Figure 1).

Three-dimensional images collected in the HM mode were selected again, and the 3DQ advanced post-processing 

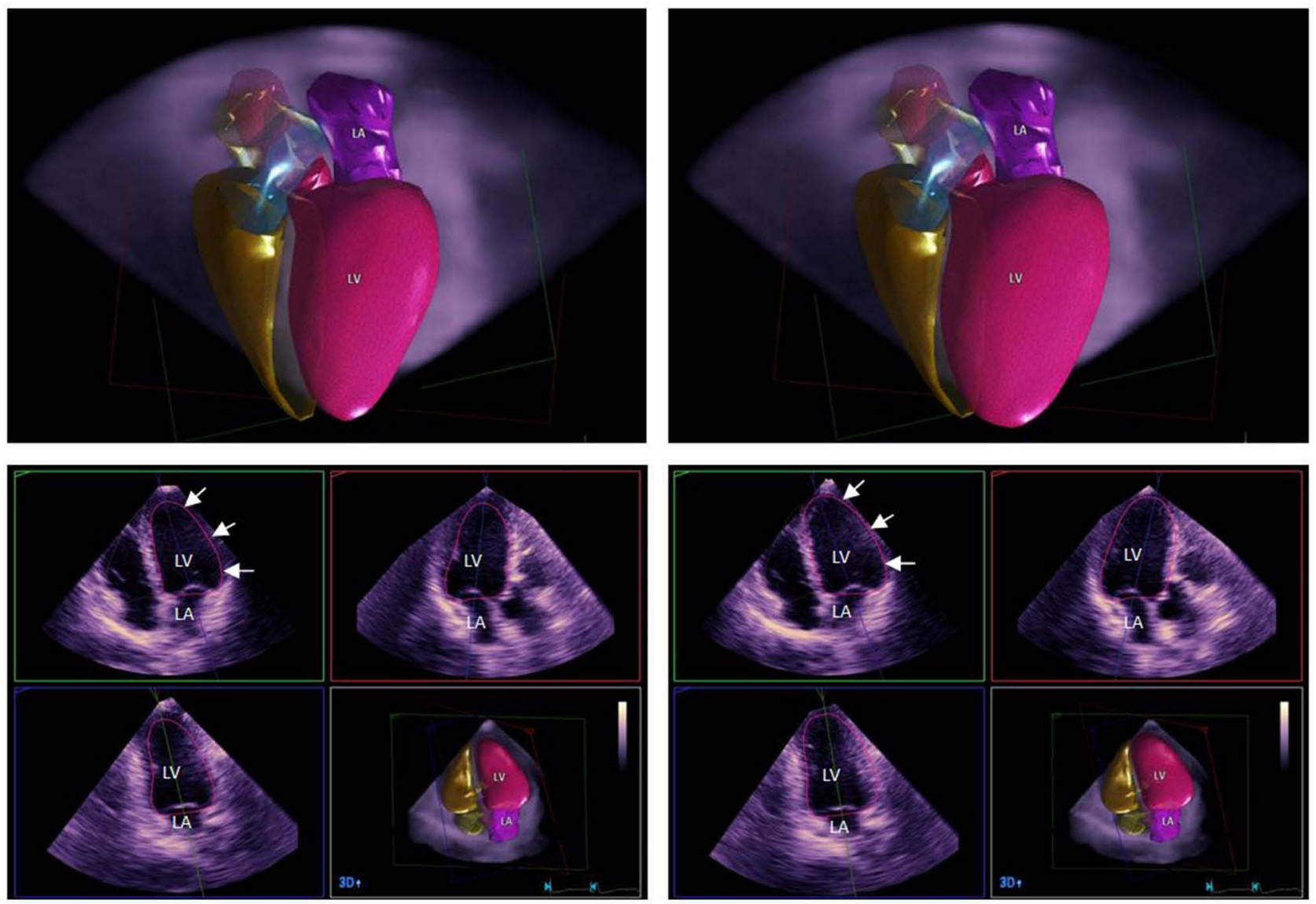

Figure I Different parameter settings of HM 3DE to evaluate left ventricular function. Left: $E D$ and $E S=40$ and 20 , right: $E D$ and ES $=100$ and 50 . Arrows: different ED and ES settings to trace the endocardium of the LV.

Abbreviations: LV, left ventricle; LA, left atrium.

software was started. The end systole and diastole were recognized by the software by marking five reference points, ie, the ventricular septum, lateral wall, anterior wall, inferior wall of the mitral valve annulus, and apex, and outlining the endocardium (which could be manually edited if necessary). The LVEDV, LVESV, LVEF, and LAV MAX were obtained (see Figure 2). The time to measure the LVEF using the HM method and the 3D method was recorded.
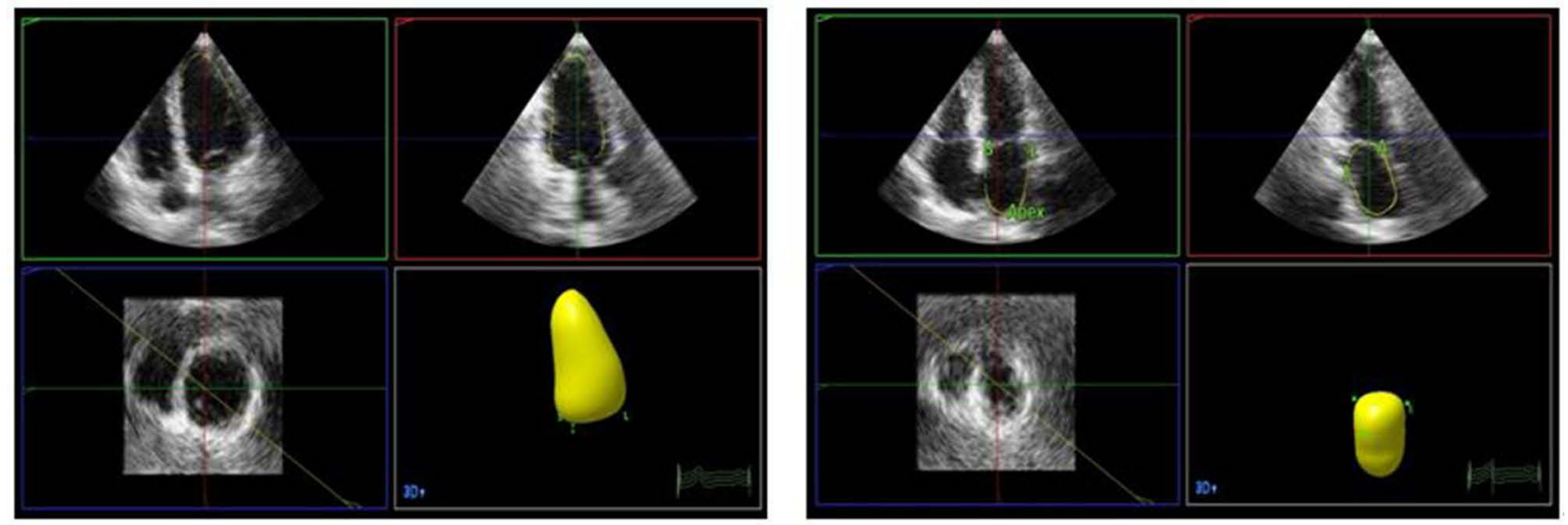

Figure 2 Conventional 3DE to evaluate left ventricular function. Left: 3D trace of the endocardium of the left ventricle, and right: 3D trace of the endocardium of the left atrium. 


\section{Inter-Observer, Intra-Observer, and Test-} Retest Reproducibility

The reproducibility of the LVEDV, LVESV, LVEF, and $\mathrm{LAV}_{\mathrm{MAX}}$ using the $\mathrm{HM}$ and $3 \mathrm{D}$ method were analyzed using a randomly derived subgroup of 30 patients.

For the inter- and intra-observer reproducibility analysis, two echocardiographers independently analyzed the same HM and 3DE loops, and one of them re-analyzed the same loops four weeks later.

For the test-retest reproducibility analysis, after the initial dataset was obtained, the echocardiographer removed the probe and repositioned the transducer five minutes later to obtain another dataset.

\section{Statistical Analysis}

The SPSS 23.0 and MedCalc software were used for statistical analysis. The measurement data were displayed as mean \pm standard deviation. The Kolmogorov-Smirnov test was used to test for a normal distribution; the paired $t$-test was used for comparisons between the two groups; the correlation between the HM and 3D method was analyzed by linear regression with Pearson correlation coefficients; the Bland-Altman test was used for the agreement analysis; and the intraclass correlation coefficient (ICC) was used for the reproducibility test, with ICC < 0.4 indicating poor reproducibility, ICC of $0.4-0.75$ indicating good reproducibility, and ICC $>0.75$ indicating very good reproducibility. $\mathrm{P}<0.05$ was considered statistically significant.

\section{Results}

A total of 113 patients were enrolled, and their datasets were analyzed. The patients included 53 males and 60 females aged 18-88 years, with an average of 51.9 years. Their baseline characteristics are presented in Table 1.

\section{Correlation Analysis of Left Ventricular Systolic Function Measured by the HM and 3D Methods}

Various settings were used to measure the different indicators of left ventricular systolic function. When comparing the $\mathrm{HM}$ and $3 \mathrm{D}$ method, the measurement
Table I Baseline Demographic Characteristics

\begin{tabular}{|l|l|}
\hline Characteristic & Value \\
\hline Age (years) & $45.91 \pm 13.83$ \\
Female gender, $\mathrm{n}(\%)$ & $60(53.10 \%)$ \\
Height $(\mathrm{cm})$ & $165.17 \pm 15.42$ \\
Weight $(\mathrm{kg})$ & $62.37 \pm 13.55$ \\
Heart Rate(beats $/ \mathrm{min})$ & $67.56 \pm 8.31$ \\
BMI $\left(\mathrm{kg} / \mathrm{m}^{2}\right)$ & $21.25 \pm 3.19$ \\
Systolic blood pressure $(\mathrm{mmHg})$ & $115.24 \pm 16.71$ \\
Diastolic blood pressure $(\mathrm{mmHg})$ & $80.5 \pm 10.97$ \\
\hline
\end{tabular}

Abbreviation: BMI, body mass index.

of LVEF showed a good correlation, with ED and ES = 70 and 30 and 70 and 40 showing the best correlation (r $=0.92$ ), and $\mathrm{ED}$ and $\mathrm{ES}=74$ and 68 showing the worst correlation $(r=0.82)$; the measurement of LVEDV had the best correlation when ED $=40(\mathrm{r}=0.75)$ and the worst when ED $=100(\mathrm{r}=0.58)$; and the measurement of LVESV had the best correlation when ES $=20(\mathrm{r}=$ $0.85)$ and the worst when ES $=68(\mathrm{r}=0.77$; see Figure 3 and Table 2).

\section{Agreement Analysis of Left Ventricular Systolic Function Measured by the HM and 3D Methods}

The agreement between the results of the 3D method and the HM method using different border settings was calculated. The level of agreement was best to LVEF when ED and $\mathrm{ES}=70$ and $30(\mathrm{BIAS}=0.12 \mathrm{~mL}, \mathrm{P}>0.05)$ and worst when $\mathrm{ED}$ and $\mathrm{ES}=74$ and $68(\mathrm{BIAS}=-14.85 \mathrm{~mL}, \mathrm{P}<$ 0.05 ; see Figures 4 and 5). For LVEDV, the agreement was best when $\mathrm{ED}=40$ (BIAS $=19.62 \mathrm{~mL}, \mathrm{P}<0.05)$ and worst when $\mathrm{ED}=100(\mathrm{BIAS}=61.22 \mathrm{~mL}, \mathrm{P}<0.05$; see Figures 4 and 5); and for LVESV, the agreement was best when $\mathrm{ES}=20(\mathrm{BIAS}=8.67 \mathrm{~mL}, \mathrm{P}<0.05)$ and worst when $\mathrm{ES}=68$ (BIAS $=30.00 \mathrm{~mL}, \mathrm{P}<0.05$; see Figure 6 and Table 2).

\section{Comparison of the Assessment of Left Ventricular Diastolic Function (LAV $\mathrm{LAX}_{\text {M }}$ ) Using the HM and 3D Methods}

Different border settings for HM did not affect $L_{A V}$ MAX. There was a good correlation between the HM and 3D method measurements for $\operatorname{LAV}_{\text {MAX }}(r=0.70)$. However, 

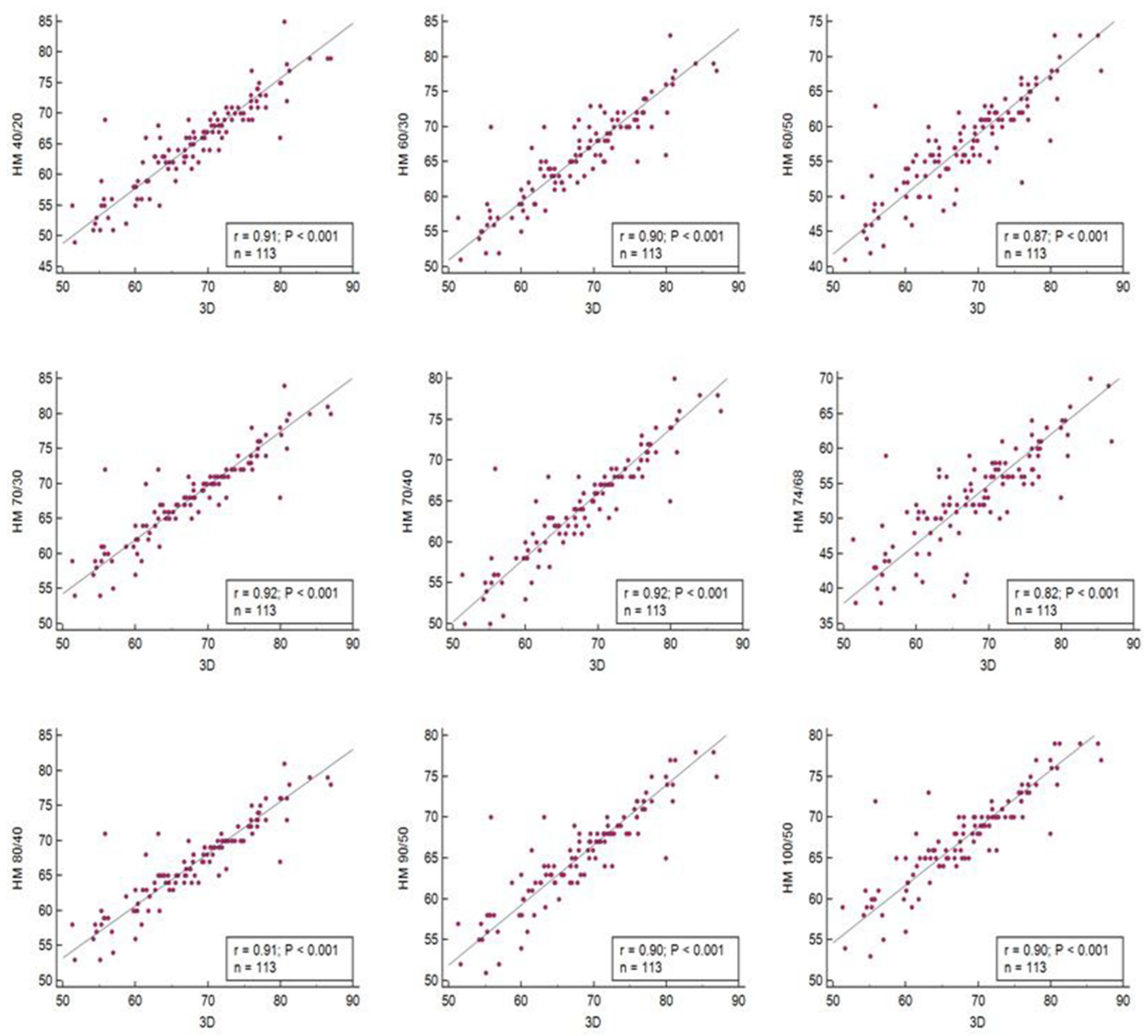

Figure 3 Correlation analysis of different HM border settings and the conventional 3DE assessment of the left ventricular ejection fraction. Abbreviations: HM 3DE, HeartModel A.I 3DE; 3DE, conventional 3DE.

the conditions for agreement were not satisfied (BIAS = $17.61 \mathrm{~mL}, \mathrm{P}<0.05$; see Table 3).

\section{Comparison of the Assessment of Reproducibility of the HM and 3D Methods}

In terms of intra-observer reproducibility, the HM software requires no human calculations; therefore, there was no variability in any of the parameters when the HM measurements were taken by the same echocardiographer with the same HM loops at different time points, and the HM method outperformed the 3D method in terms of intra-observer reproducibility. The HM method also outperformed the 3D method in terms of inter-observer and test-retest reproducibility, which were similar to each other, as a different dataset was being used (see Table 4).

\section{Comparison of the Measurement Time of the HM and 3D Methods}

The post-processing time of the HM method was significantly less than the 3D method ( $<<0.05$; see Table 5). 


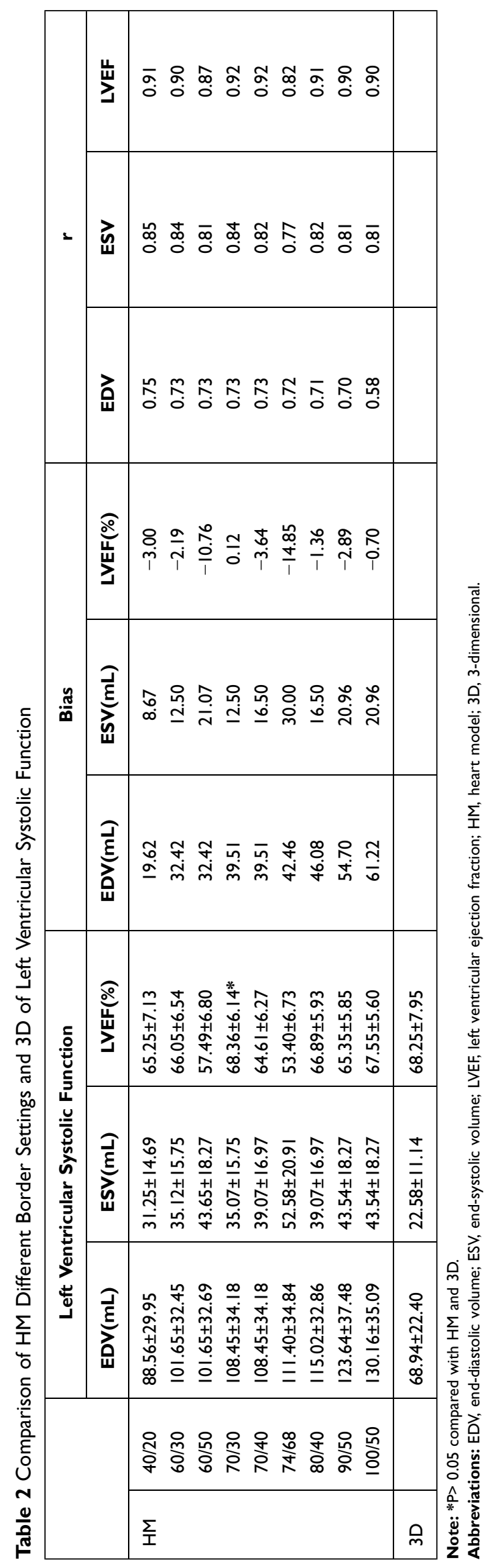

\section{Discussion}

\section{Advancements in 3DE Research}

Since the emergence of ultrasound imaging of the mitral valve in 1953, with the rapid development of computer technology and the doubling of the number of transistors per square inch in integrated circuits, $3 \mathrm{DE}$ emerged in the 21 st century, its resolution gradually improved, and progress was made. The application of echocardiography to measure heart function has always been of the utmost importance to clinicians, with ultrasound doctors pursuing the goal of accurately measuring heart function. Continuous improvements are being sought in the accuracy and reproducibility of cardiac function assessments, from the traditional M-mode echocardiography to the 2D biplane Simpson method and the 3DE. ${ }^{10}$ Through the acquisition of full-volume images, conventional 3DE achieves a revolutionary change from traditional methods that rely on geometric assumptions to estimate the volume of the heart chamber by directly measuring the volume of the true heart chamber. It avoids the problems of foreshortened views and measurement errors in assessing irregular heart chambers and performs a more accurate assessment of cardiac function. ${ }^{11}$ In 2015, the European Association of Echocardiography and the American Society of Echocardiography recommended that where possible, 3DE should be used to evaluate the left heart function. ${ }^{12}$ However, 3DE is complex, specialized training is required, and it is time consuming to use. Therefore, given the high number of disease sources, the large population of China, and the severe workload of echocardiographers in China, for more than 10 years, $3 \mathrm{DE}$ has primarily been used for research rather than in clinical practice. $^{4,5}$ Therefore, HM 3DE may have a chance to bridge the gap between the available technology and clinical practice. In recent studies, HM 3DE has been used to evaluate left ventricular systolic function in multiple populations ${ }^{6,13,14}$ and in patients with various heart diseases, ${ }^{15}$ but there is no unified standard for the border parameter settings, and there are few studies on Asian populations. Therefore, this study is important and worthy of discussion.

\section{Experimental Design}

In this study, we used conventional $3 \mathrm{DE}$ as a comparison, which has been proven to have a good correlation with CMR. It has been reported that the 

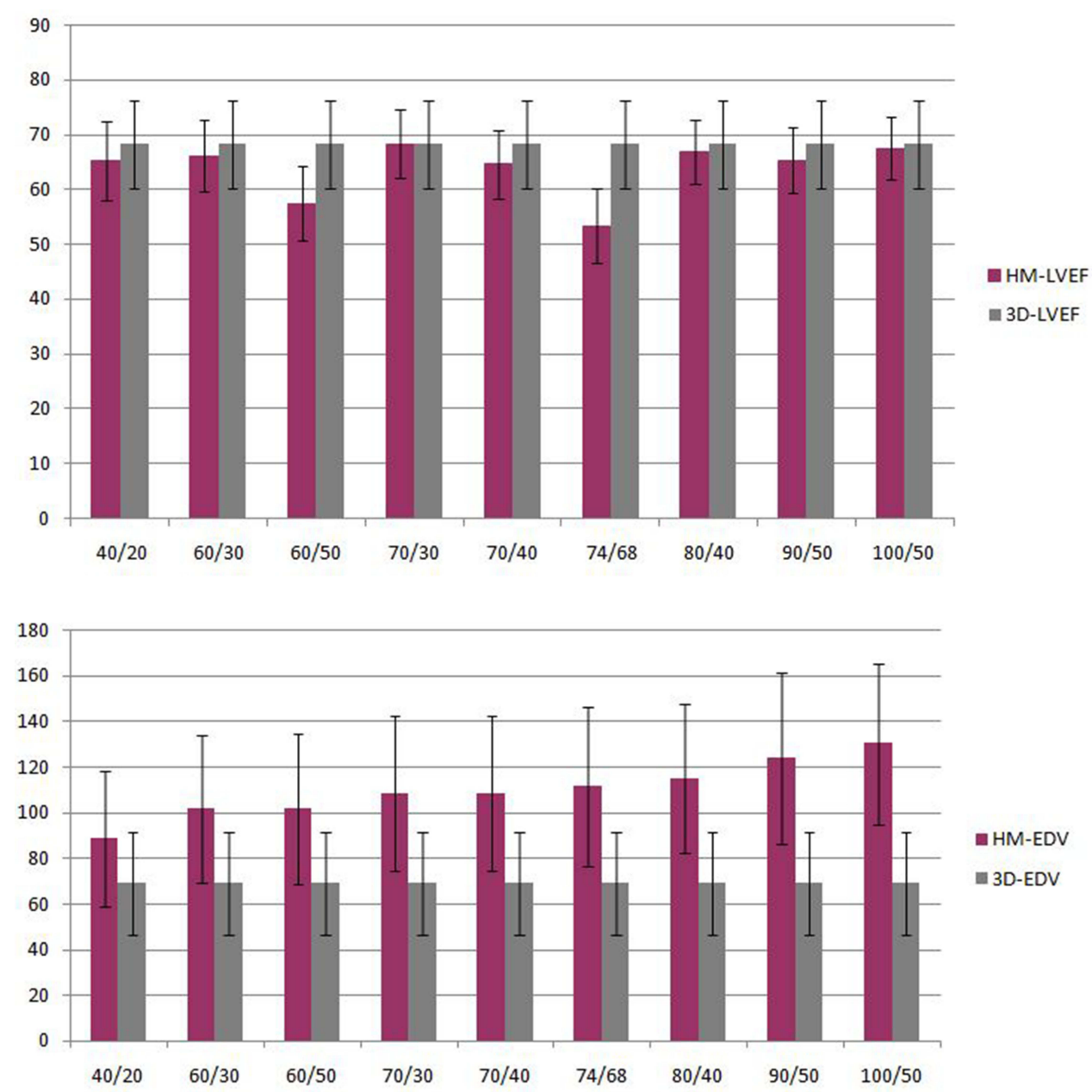

Figure 4 Up: Different parameter settings of HM to evaluate LVEF compared with conventional 3DE. Down: Different parameter settings of HM to evaluate EDV (mean) compared with conventional 3DE.

Abbreviations: LVEF, left ventricular ejection fraction; EDV, end-diastolic volume; HM, HeartModel A.I.; 3D, three-dimensional; HM 3DE, HeartModel A.I 3DE; 3DE, conventional 3DE.

border parameter settings used in HM 3DE are inconsistent, and there is no relevant guideline or consensus to recommend the optimal settings. However, previous literature on the use of different border parameter settings revealed little correlation between them and the measurement results of the EF and some correlation with the measurement of the heart cavity volume. ${ }^{9}$ Therefore, this study applied different border parameter settings based on the literature review, ${ }^{9,16}$ the recommendations of the machine manufacturer, and the difference in the heart chamber size and wall thickness between the Chinese population and other populations and made a comparative analysis. In addition, due to the improvement in the diagnosis and treatment of heart failure, the assessment of heart function has become more refined. The accurate and reproducible measurement of heart function provides an important reference value for clinical diagnosis and treatment. Therefore, we also conducted a reproducibility assessment.

\section{Analysis of the Results}

When considering the agreement analysis, a setting of $\mathrm{ED}$ and $\mathrm{ES}=70$ and 30 showed the best agreement with conventional $3 \mathrm{DE}$, and the difference in the readings was not statistically significant (BIAS $=0.12 \mathrm{~mL}$, $\mathrm{P}>0.05)$, indicating that it is feasible to use the HM method to evaluate left ventricular systolic function. However, when measuring LVEDV and LVESV, a setting of ED and ES $=40$ and 20 showed the best agreement with the 3D method. Although LVEF measured by $3 \mathrm{DE}$ in the assessment of left ventricular 

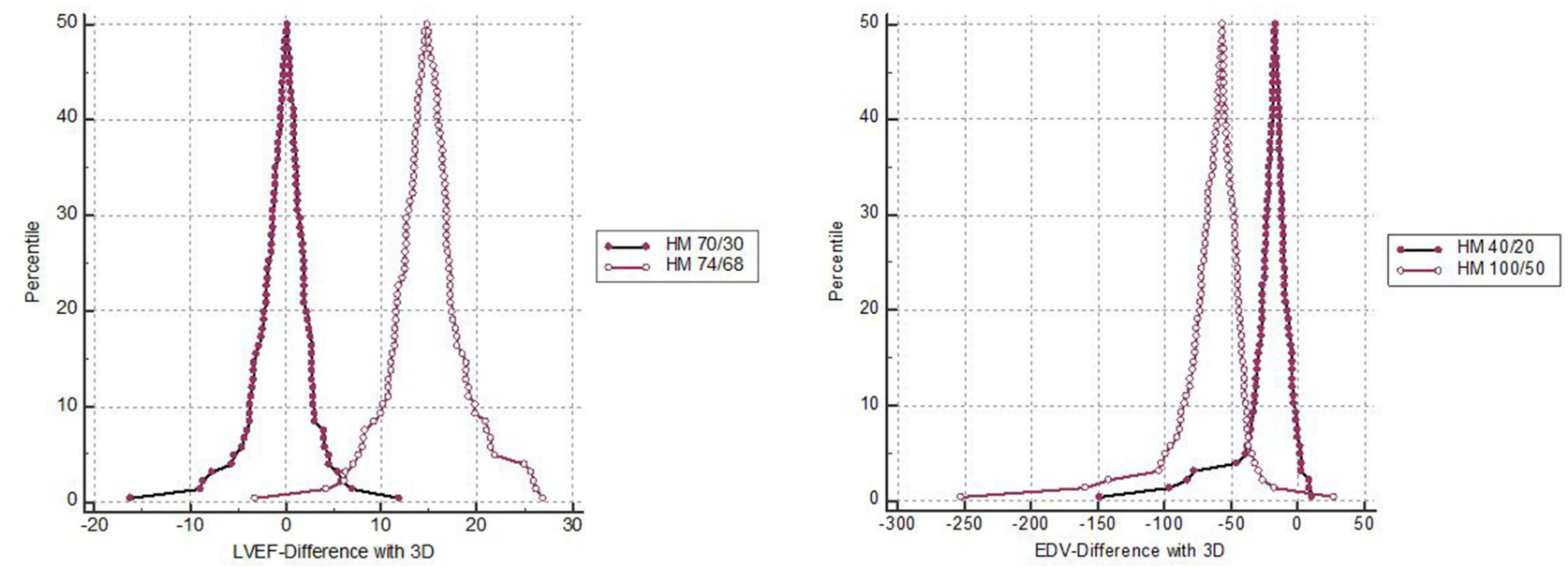

Figure 5 Left: Comparison of the agreement of LVEF between different HM border settings and conventional 3DE. ED and ES $=70$ and 30 was much better than ED and ES $=74$ and 68. Right: Comparison of the agreement of EDV between different HM border settings and conventional 3DE. ED and ES $=40$ and 20 was much better than ED and $\mathrm{ES}=100$ and 50 .

Abbreviations: LVEF, left ventricular ejection fraction; EDV, end-diastolic volume; HM, HeartModel A.l.; 3D, three-dimensional; HM 3DE, HeartModel A.I 3DE; 3DE, conventional 3DE.

systolic function showed a good correlation with CMR, due to different measurement principles and the way in which the endocardium is displayed, the 3D method may underestimate left ventricular volume, which is consistent with the results of previous studies. ${ }^{6,14,15}$ In addition, the good correlation and poor agreement of $\mathrm{LAV}_{\text {MAX }}$ measured by the $3 \mathrm{D}$ and HM methods may also be related to the underestimation of the $3 \mathrm{D}$ method in volume measurements.

When considering reproducibility, it was clear that the results of measurements using the HM method were more reproducible than from the 3D method, which reveals the advantages of the HM method in automated tracing. Three-dimensional echocardiography is a semi-automatic measurement, which requires manual point setting and manual adjustment of the endocardium, while the HM method is an automatic measurement, which does not require human intervention.

When considering measurement time, the HM method took less time than the 3D method, which is consistent with previous studies. ${ }^{6}$

In summary, we concluded that HM 3DE exhibits a high degree of repeatability, and a border setting of $\mathrm{ED}$ and $\mathrm{ES}=70$ and 30 is the most suitable setting for studying the left ventricular function in the Chinese population. However, in assessing the volume of heart chamber, there was a possibility underestimation when using the $3 \mathrm{D}$ method, which is supported by previous studies (except in pediatric patients[13]), and this requires further exploration. In addition, the measurements from the HM method showed the best agreement with the 3D method when ED and ES $=40$ and 20, but the difference was still statistically significant. The results are therefore inconclusive as to whether a setting of $40 / 20$ or $70 / 30$ is most appropriate for the assessment of left ventricular volume. Supplementary studies should be conducted in this area to determine the level of agreement of the left heart volume measured using the HM method and CMR.

\section{Limitations}

In this study, the feasibility of HM 3DE to evaluate left ventricular function was discussed in more detail based on previous research. There are some limitations that require further studies to be conducted. First, the study subjects were people without a history of heart disease; arrhythmia, such as atrial fibrillation; or changes in heart size, such as heart failure. However, HM can be used to analyze images from a single cardiac cycle, and the HM database consists of a large number of different 3D heart morphologies that have promising potential for evaluating abnormal hearts. Second, this study was single-center study with a small sample size. The sample size needs to be increased, and repeat research needs to be conducted to ensure more convincing results and make HM 3DE feasible for use in clinical practice. Finally, this study only verified a few 

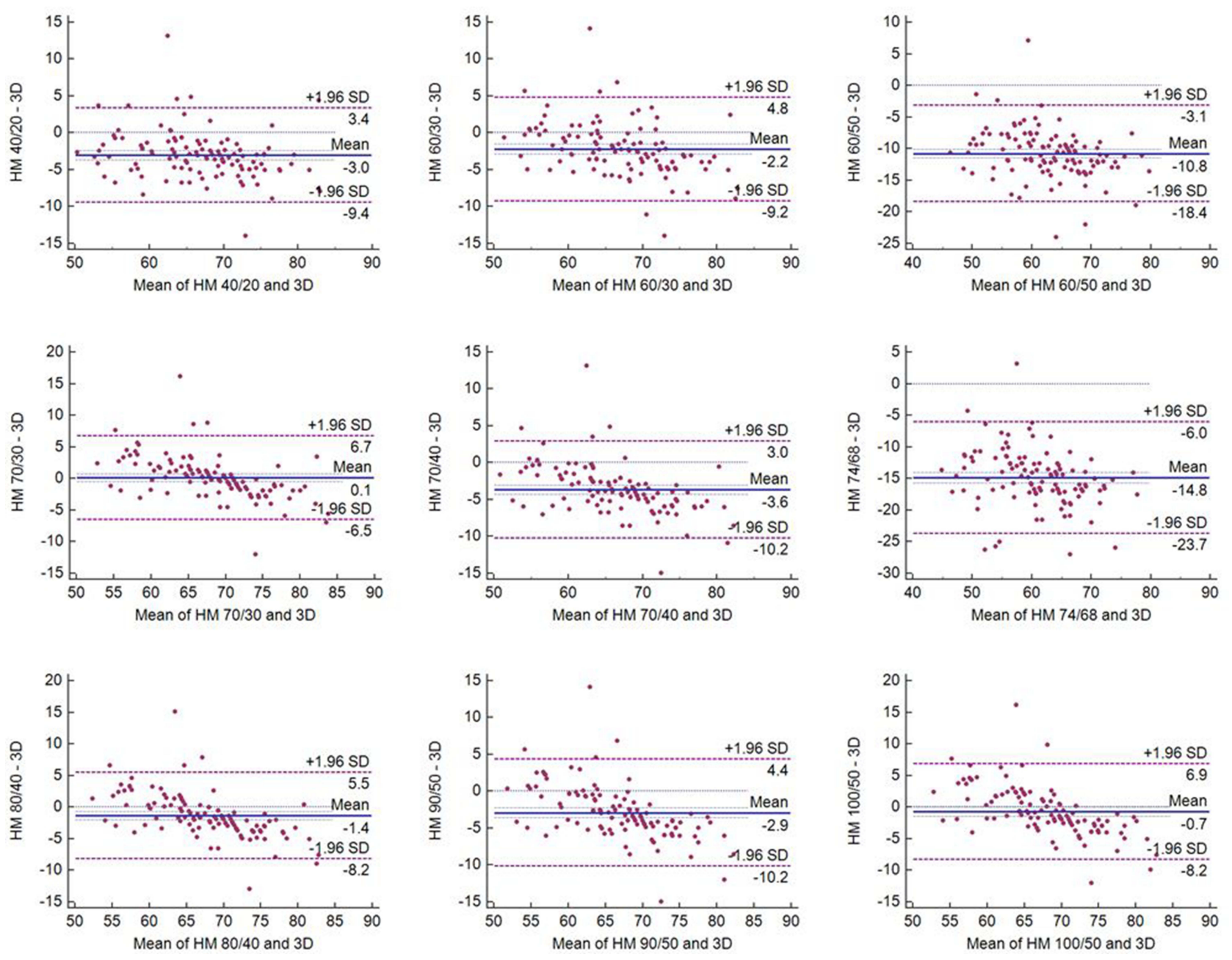

Figure 6 Agreement analysis of different HM border settings and conventional 3DE assessment of left ventricular ejection fraction. Abbreviations: HM 3DE, HeartModel A.I 3DE; 3DE, conventional 3DE.

indicators of left heart function. With the continuous development of artificial intelligence technology, it will become possible to take automatic measurements of many more functions. We look forward to these advancements. Also, plausibility of the study could be increased with the larger study population. And only patients without cardiac diseases and with good acoustic window were enrolled into the study which does not even remotely reflects the reality of echocardiographic room. In clinical practice accurate LVEF assessment is

Table 3 Comparison of HM and 3D of LAVmax

\begin{tabular}{|l|c|c|c|}
\hline & LAV $_{\text {MAX }}(\mathrm{mL})$ & Bias & $\boldsymbol{r}$ \\
\hline HM & $53.86 \pm 22.48$ & 17.61 & 0.70 \\
3D & $36.25 \pm 15.06$ & & \\
\hline
\end{tabular}

Abbreviations: HM, heart model; 3D, 3-dimensional; LAV MAX, maximal left atrium volume. particularly essential with impaired LV systolic function.

\section{Conclusion}

A setting of ED and ES $=70$ and 30 shows the best correlation between HM 3DE and conventional 3DE in the Chinese population. Although HM 3DE may still show some discrepancies when compared with CMR, its remarkable rapidity

Table 4 Reproducibility (Intraclass Correlation Coefficient, ICC)

\begin{tabular}{|l|c|c|c|c|c|c|}
\hline \multirow{2}{*}{} & \multicolumn{2}{|c|}{ Inter-Observer } & \multicolumn{2}{c|}{ Intra-Observer } & \multicolumn{2}{c|}{ Test-Retest } \\
\cline { 2 - 7 } & 3D & HM & 3D & HM & 3D & HM \\
\hline LVEDV & 0.79 & 1.00 & 0.82 & 1.00 & 0.80 & 0.95 \\
LVESV & 0.80 & 1.00 & 0.86 & 1.00 & 0.83 & 0.96 \\
LVEF & 0.82 & 1.00 & 0.85 & 1.00 & 0.83 & 0.98 \\
LAV $_{\text {MAX }}$ & 0.80 & 1.00 & 0.82 & 1.00 & 0.80 & 0.97 \\
\hline
\end{tabular}


Table 5 Comparison of HM and 3D Assessment of Time

\begin{tabular}{|l|c|c|}
\hline & 3D(sec) & HM(sec) \\
\hline LVEF & $50.16 \pm 8.51$ & $22.37 \pm 2.65^{*}$ \\
LAV $_{\text {MAX }}$ & $30.47 \pm 7.22$ & $22.37 \pm 2.65^{*}$ \\
\hline
\end{tabular}

Note: $* \mathrm{P}<0.05$ compared with $\mathrm{HM}$ and 3D.

Abbreviations: LVEF, left ventricular ejection fraction; $L A V_{M A X}$, maximal left atrium volume; HM, Heart model; 3D, 3-dimensional.

and reproducibility give it great potential in daily application for echocardiographic examination.

\section{Data Sharing Statement}

The datasets used and/or analysed during the current study available from the corresponding author on reasonable request.

\section{Ethics Approval and Consent to Participate}

This study was conducted in accordance with the declaration of Helsinki.This study was conducted with approval from the Ethics Committee of Hebei General Hospital.A written informed consent was obtained from all participants.

\section{Consent for Publication}

Consent for publication was obtained from every individual whose data are included in this manuscript.

\section{Funding}

This study was supported by Grant 20190406 from Hebei Health and Family Planning Commission, 203777105D from Science and Technology Department of Hebei province.

\section{Disclosure}

None of the authors have any financial disclosure or conflicts of interest.

\section{References}

1. Ponikowski P, Voors AA, Anker SD, et al. Developed with the special contribution of the Heart Failure Association (HFA) of the ESC. Eur J Heart Fail. 2016;18(8):891-975.

2. Altman M, Bergerot C, Aussoleil A, et al. Assessment of left ventricular systolic function by deformation imaging derived from speckle tracking: a comparison between 2D and 3D echo modalities. Eur Heart J Cardiovasc Imaging. 2014;15(3):316-323. doi:10.1093/ehjci/ jet103
3. Sugeng L, Mor-Avi V, Weinert L, et al. Quantitative assessment of left ventricular size and function: side-by-side comparison of real-time three-dimensional echocardiography and computed tomography with magnetic resonance reference. Circulation. 2006;114 (7):654-661. doi:10.1161/CIRCULATIONAHA.106.626143

4. Muraru D, Badano LP, Ermacora D, Piccoli G, Iliceto S. Sources of variation and bias in assessing left ventricular volumes and dyssynchrony using three-dimensional echocardiography. Int $J$ Cardiovasc Imaging. 2012;28:1357-1368. doi:10.1007/s10554-011-9985-0

5. Tsang W, Kenny C, Adhya S, et al. Interinstitutional measurements of left ventricular volumes, speckle-tracking strain, and dyssynchrony using three-dimensional echocardiography. J Am Soc Echocardiogr. 2013;26(11):1253-1257. doi:10.1016/j.echo.2013.07.023

6. Tsang W, Salgo IS, Medvedofsky D, et al. Transthoracic 3D echocardiographic left heart chamber quantification using an automated adaptive analytics algorithm. JACC Cardiovasc Imaging. 2016;9 (7):769-782. doi:10.1016/j.jcmg.2015.12.020

7. Medvedofsky D, Mor-Avi V, Amzulescu M, et al. Three-dimensional echocardiographic quantification of the left-heart chambers using an automated adaptive analytics algorithm: multicentre validation study. Eur Heart J Cardiovasc Imaging. 2018;19(1):47-58. doi:10.1093/ehjci/ jew328

8. D'Elia N, Appadurai V, Mallouhi M, Ng J, Marwick T, Wahi S. Comparison of $3 \mathrm{D}$ echocardiographic-derived indices using fully automatic left ventricular endocardial tracing (heart model) and semiautomatic tracing (3DQ-ADV). Echocardiography. 2019;36:20 57-2063. doi:10.1111/echo.14502

9. Barletta V, Hinojar R, Carbonell A, et al. Three-dimensional full automated software in the evaluation of the left ventricle function: from theory to clinical practice. Int $J$ Cardiovasc Imaging. 2018;34:1205-1213. doi:10.1007/s10554-018-1336-y

10. Spitzer E, Ren B, Zijlstra F, Mieghem NMV, Geleijnse ML. The role of automated $3 \mathrm{~d}$ echocardiography for left ventricular ejection fraction assessment. Card Fail Rev. 2017;3:97-101. doi:10.15420/cfr.2017:14.1

11. Mor-Avi V, Yodwut C, Jenkins C, et al. Real-time 3D echocardiographic quantification of left atrial volume: multicenter study for validation with CMR. JACC Cardiovasc Imaging. 2012;5 (8):769-777. doi:10.1016/j.jcmg.2012.05.011

12. Lang RM, Badano LP, Mor-Avi V, Afilalo JU. Recommendations for cardiac chamber quantification by echocardiography in adults: an update from the American Society of Echocardiography and the European Association of Cardiovascular Imaging. $J \mathrm{Am}$ Soc Echocardiogr. 2015;28(1):1-39.e14. doi:10.1093/ehjci/jev014

13. Carvajal-Rivera JJ, Lopez-Quintero JC, Gonzalez-Menchen C, de Agustin JA, Macaya C, Perez de Isla L. Left ventricular volumes and ejection fraction quantification using an automated three-dimensional adaptive analytic echocardiographic algorithm in pediatric population. Echocardiography. 2018;35:1827-1834. doi:10.1111/echo.14126

14. Luo XX, Fang F, So HK, Liu C, Yam MC, Lee AP. Automated left heart chamber volumetric assessment using three-dimensional echocardiography in Chinese adolescents. Echo Res Pract. 2017;4:53-61. doi:10.1530/ERP-17-0028

15. Otani K, Nakazono A, Salgo IS, Lang RM, Takeuchi M. Threedimensional echocardiographic assessment of left heart chamber size and function with fully automated quantification software in patients with atrial fibrillation. $J \mathrm{Am}$ Soc Echocardiogr. 2016;29:955-965. doi:10.1016/j.echo.2016.06.010

16. Levy F, Dan Schouver E, Iacuzio L, et al. Performance of new automated transthoracic three-dimensional echocardiographic software for left ventricular volumes and function assessment in routine clinical practice: comparison with 3 Tesla cardiac magnetic resonance. Arch Cardiovasc Dis. 2017;110(11):580-589. doi:10.10 16/j.acvd.2016.12.015 


\section{Publish your work in this journal}

The International Journal of General Medicine is an international, peer-reviewed open-access journal that focuses on general and internal medicine, pathogenesis, epidemiology, diagnosis, monitoring and treatment protocols. The journal is characterized by the rapid reporting of reviews, original research and clinical studies across all disease areas. The manuscript management system is completely online and includes a very quick and fair peer-review system, which is all easy to use. Visit http://www.dovepress.com/ testimonials.php to read real quotes from published authors.

Submit your manuscript here: https://www.dovepress.com/international-journal-of-general-medicine-journal 